\title{
PROJETO ECONÔMICO DE CARTAS ADAPTATIVAS PARA MONITORAMENTO DE PROCESSOS
}

\section{GESTÃO \\ $\&$ \\ PRODUÇÃO}

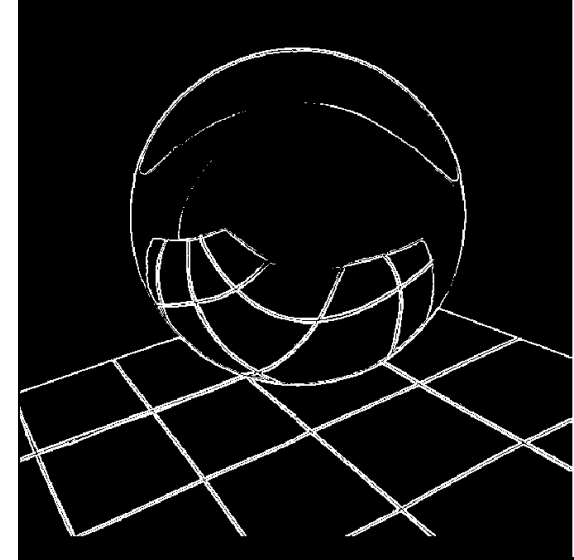

v.9, n.1, p.17-31, abr. 2002
Renato Michel

Flávio S. Fogliatto

Departamento de Engenharia de Produção e Transportes

Universidade Federal do Rio Grande do Sul

Porto Alegre - RS

E-mails: nrmichel@portoweb.com.br

ffogliatto@ppgep.ufrgs.br

\section{Resumo}

Cartas de controle estatístico vêm sendo utilizadas com sucesso no monitoramento do desempenho de processos industriais. Nos últimos anos, diversas modificações nas cartas de Shewhart foram propostas na literatura. Tais modificações visam adaptar as cartas de controle ao monitoramento de processos com características especiais; entre elas, destacam-se as cartas adaptativas de controle. Apesar de apresentarem vantagens sobre as cartas de Shewhart, sinalizando, de maneira mais rápida, situações anômalas no comportamento dos processos, aspectos práticos relacionados à implementação de cartas adaptativas são pouco explorados na literatura. Neste artigo, propõe-se um modelo econômico para o projeto de cartas adaptativas. O modelo proposto é aplicado em um estudo de caso, no monitoramento de um processo de usinagem de peças para automóveis. No estudo de caso, o significado e a forma de determinação dos parâmetros do modelo econômico são detalhados. Esquemas adaptativos e tradicionais de controle de processo são comparados quanto a seus custos operacionais.

Palavras-chave: cartas adaptativas de controle, modelo econômico para cartas de controle, controle estatístico de processo, indústria automotiva.

\section{Introdução}

Cartas de controle (CCs) estatístico são ferramentas de monitoramento do desempe- nho de processos. Elas utilizam como dados de entrada medições de variáveis que influenciam na qualidade dos itens manufaturados. Medições são realizadas em pontos espaçados no tempo e 
registradas nas cartas; este registro resulta em gráficos temporais que apresentam os valores de medição da variável no eixo vertical, e os pontos no tempo nos quais as medições são efetuadas, no eixo horizontal. A cada medição, compara-se o resultado obtido com limites de controle: medições fora dos limites indicam a presença de causas especiais de variabilidade, anômalas ao processo, que prejudicam a qualidade do produto manufaturado. Uma vez identificadas as causas especiais, pode-se atuar sobre elas, melhorando continuamente a qualidade do produto. CCs permitem a redução sistemática da variabilidade nas características de qualidade (CQs) do produto, representadas pelas variáveis monitoradas nas cartas. Assim, melhora-se a qualidade intrínseca, a produtividade, a confiabilidade e o custo do que está sendo produzido.

CCs são ferramentas simples e eficazes, sendo muito utilizadas na prática do controle de qualidade. A intensa utilização das CCs na indústria tem motivado estudos que buscam seu aperfeiçoamento. Na última década, uma nova classe de CCs, as cartas adaptativas de controle (CACs), tem sido objeto de pesquisa na área da Engenharia da Qualidade (ver TAGARAS, 1998, para uma extensa compilação de trabalhos publicados sobre CACs; ver também COSTA, 1998, para uma referência em português sobre o tema). Uma CC é dita adaptativa se ao menos um de seus parâmetros - intervalo de amostragem, tamanho da amostra ou coeficiente dos limites de controle - variar durante o monitoramento do processo, como função de seu desempenho observado.

Nas CACs, o estado atual do processo é definido pelo valor da estatística amostral plotada na carta. A idéia básica das CACs é intensificar o controle sobre o processo quando pontos no gráfico se aproximarem dos limites de controle, e relaxar o controle quando os pontos estiverem próximos da linha central. A intensidade de controle é definida através de mudanças nos valores dos parâmetros da carta.

As CACs apresentam vantagens, considerando-se aspectos estatísticos e econômicos, em relação às CCs tradicionais. Sob o ponto de vista estatístico, há uma redução no tempo médio até a sinalização da ocorrência de uma causa especial, como consequiência do aumento na taxa de amostragem (tomada de amostras maiores e/ou mais freqüentes) quando a carta apresentar evidências de que o processo está fora de controle. Sob o ponto de vista econômico, há uma redução nos custos relacionados ao controle do processo, causada pela redução na taxa de amostragem quando a carta indicar processo sob controle. Como conseqüência do melhor desempenho estatístico das CACs, verifica-se, também, uma redução nos custos relacionados à produção de itens de má qualidade, diretamente vinculada à redução do tempo até a sinalização das causas especiais.

Neste artigo, apresenta-se um modelo econômico para o projeto de CACs para o monitoramento de médias de CQs de interesse. Modelos econômicos permitem determinar o tamanho de amostra e frequiência de amostragem a ser adotados em CACs, bem como seus limites de controle e advertência. O objetivo principal é desenvolver subsídios que possibilitem a implantação prática de CACs em meios de manufatura repetitiva. Para tanto, o modelo proposto é aplicado a um estudo de caso, no projeto de uma CAC para monitoramento da concentricidade de uma autopeça, usinada em torno mecânico do tipo CNC (Computerized Numeric Control). O modelo econômico aqui proposto propõe uma adaptação ao modelo de DAS et al. (1997).

Modelos econômicos para CCs demandam informações sobre um grande número de parâmetros de entrada. Parte desses parâmetros diz respeito a custos de monitoramento do processo mediante cenários distintos; por exemplo, processo sob controle ou sob incidência de causas especiais. Outros parâmetros estão relacionados a tempos; por exemplo, tempo médio necessário para coletar uma amostra ou para identificar uma causa especial. No geral, modelos econômicos apresentados na literatura são desenvolvimentos essencialmente teóricos, 
validados por simulações computacionais (PARKHIDEH \& CASE, 1989 e DAS et al., 1997, entre outros), o que resulta em dúvidas quanto à determinação dos parâmetros do modelo. O presente artigo traz uma importante contribuição nesse sentido. A partir de um estudo de caso, aspectos relacionados à determinação prática de parâmetros do modelo proposto são detalhados. Além disso, utilizando dados do estudo de caso, o artigo confronta os custos associados ao monitoramento tradicional e adaptativo de uma CQ, utilizando CCs. Foi possível verificar, na prática, a superioridade das CACs sobre as CCs tradicionais, em particular na detecção de pequenos desvios na média da CQ sob monitoramento. Este resultado corrobora afirmações feitas por RUNGER \& PIGNATIELLO (1991) e REYNOLDS et al. (1996), entre outros, baseadas em simulações computacionais.

A estrutura deste artigo é a seguinte. A seção 2 introduz CCs tradicionais, o conceito de CACs e seus princípios básicos de operação, e as CACs para médias, foco deste trabalho. A seção 3 traz o modelo econômico proposto para as CACs para médias. A seção 4 apresenta a aplicação do modelo econômico ao estudo de caso. A conclusão encerra o artigo na seção 5.

\section{Cartas Adaptativas de Controle de Processo}

A apresentação das cartas adaptativas de controle (CACs) para o monitoramento de médias utiliza conceitos relacionados a cartas de controle (CCs) tradicionais, apresentados a seguir.

O objetivo das CCs é monitorar a variabilidade existente nos processos, distinguindo causas comuns (causadoras de pequenas variações aleatórias e, via de regra, inofensivas ao processo) de causas especiais. Causas especiais devem ser identificadas e corrigidas para que o processo permaneça dentro de um padrão esperado de desempenho.

Algumas CCs monitoram CQs que podem ser medidas e expressas em uma escala contínua de valores, como, por exemplo, volume, comprimento e temperatura. Outras CCs monitoram
CQs como o número de produtos conformes ou não-conformes (defeituosos), ou o número de não-conformidades (defeitos) em uma unidade do produto. Essas cartas são conhecidas como CCs para variáveis e para atributos, respectivamente. As CCs de interesse neste trabalho são as cartas $\bar{X}$, para variáveis. As cartas $\bar{X}$ monitoram, pela média amostral, a localização da variável de interesse.

Cartas de controle, independentemente do tipo, baseiam-se nos mesmos princípios fundamentais de construção e operação. As CCs apresentam uma linha central, que representa a média da variável de interesse quando o processo está sob controle (ou livre de causas especiais) e duas outras linhas, que representam os limites de controle do processo (ver exemplo na Figura 1; PROCEP, 1999). Limites de controle são definidos de forma a compreender a maior parte dos valores da estatística amostral plotada, estando o processo sob controle. $\mathrm{O}$ processo é dito fora de controle quando um ou mais pontos da carta caírem além dos limites de controle ou apresentarem um comportamento não aleatório. Um exemplo deste comportamento pode ser uma série de pontos consecutivos de um mesmo lado da carta, acima ou abaixo da linha central, mesmo que nenhum ponto caia fora dos limites de controle. O conjunto de regras que determinam o que é um comportamento sistemático ou não-aleatório é chamado de regras de corrida (run rules), e a política de controle pode ou não considerá-las para monitorar o processo (MONTGOMERY, 1996; DAS, JAIN \& GOSAVI, 1997; TAGARAS, 1998). Pontos além dos limites de controle e com comportamento sistemático não-aleatório encontram-se assinalados (circundados) como "Causas Especiais" na Figura 1.

Considere as medições $x_{1}, x_{2}, \ldots$, de uma CQ de interesse, com média $\mu$ e desvio-padrão $\sigma$. A determinação da linha central e dos limites de controle para uma carta $\bar{X}$ segue um modelo geral, onde $\bar{x}\left(=\frac{1}{n} \sum_{i=1}^{n} x_{i}\right)$ é a estatística amostral que representa a CQ de interesse, com média 


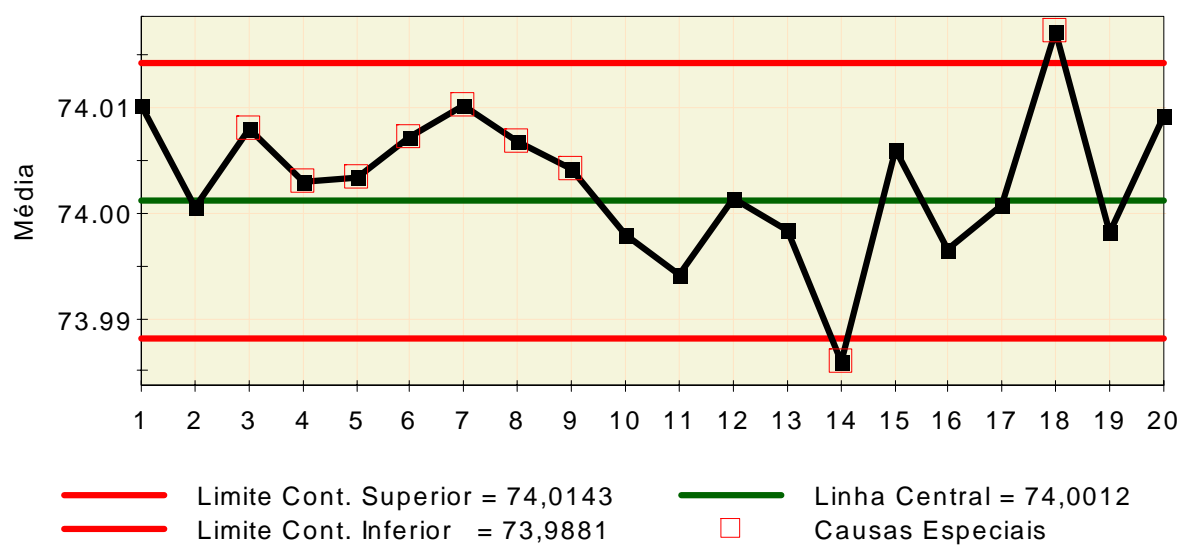

Figura 1 - Exemplo de carta de controle $\bar{X}$.

$\mu_{\bar{X}}=\mu$ e desvio-padrão $\sigma_{\bar{X}}=\frac{\sigma}{\sqrt{n}}$. Assim, o

limite de controle superior (LCS), a linha central (LC) e o limite de controle inferior (LCI) são dados por (MONTGOMERY, 1996):

$$
\begin{aligned}
& L C S=\mu+L \sigma_{\bar{X}} ; \\
& L C=\mu ; \\
& L C I=\mu-L \sigma_{\bar{X}} ;
\end{aligned}
$$

onde $L$ é a distância, em desvios-padrão da média, entre os limites de controle e a linha central. Quando a média e o desvio-padrão da CQ são desconhecidos, seus valores são estimados, a partir de amostragem preliminar, por $\overline{\bar{x}}=\sum_{i=1}^{k} \bar{x}_{i} / k$ e $\hat{\sigma}=\bar{R} / d_{2}$, respectivamente, onde $k$ indica o número de amostras utilizadas; $\overline{\bar{x}}$ é a "média das médias" das $k$ amostras; $\bar{R}$ é a média das amplitudes das $k$ amostras e $d_{2}$ é a média da amplitude relativa. Os valores de $d_{2}$ dependem de $n$ e são tabelados (MONTGOMERY, 1996).

$\mathrm{O}$ valor de $L$ mais comumente utilizado em CCs é $L=3$, o que pode ser justificado pelos bons resultados obtidos na prática. Nas CCs abordadas neste artigo, a estatística $\bar{x}$ será substituída por uma variável padronizada $z=(\bar{x}-\mu) /(\sigma / \sqrt{n})$.
Além do valor de $L$, que determina os limites de controle, é indispensável ao planejamento de uma CC a determinação do tamanho da amostra e da frequiência de amostragem. Tais parâmetros são definidos tendo em vista (i) o tamanho da mudança no processo a ser detectada pela CC e (ii) a rapidez com que se deseja detectar esta mudança. Pequenas mudanças no processo são rapidamente detectadas utilizando-se grandes amostras tomadas frequientemente, o que é, no entanto, economicamente inviável. Uma prática mais adequada é a escolha entre pequenas amostras tomadas freqüentemente (política mais utilizada pela indústria) ou grandes amostras tomadas em intervalos de tempo maiores.

A escolha dos limites de controle influencia diretamente as probabilidades de ocorrência dos erros tipo I e tipo II. O erro tipo I é caracterizado pela ocorrência de um alarme falso (sinalização incorreta da incidência de causas especiais sobre o processo). O erro tipo II é caracterizado pela não-detecção de uma situação real de processo fora de controle. Quanto mais distantes da linha central estiverem os limites de controle, menor será a probabilidade de erro tipo I, aumentando, todavia, a probabilidade de erro tipo II. Por outro lado, aproximar os limites de controle da linha central provoca uma situação inversa. 
Ao contrário das CCs tradicionais, CACs permitem parâmetros variáveis, definidos de acordo com o estado do processo, determinado pelo valor da estatística amostral na CC. Os parâmetros adaptáveis nas CACs compreendem (a) freqüência de amostragem, (b) tamanho da amostra e $(c)$ coeficiente dos limites de controle. A mudança nos valores dos parâmetros ocorre em tempo real, durante o monitoramento do processo.

O funcionamento das CACs pode ser assim descrito. Suponha uma CC com pontos plotados em uma região entre limites de advertência, mais próximos à linha de centro do que os limites usuais de controle, e os limites $3 \sigma_{\bar{X}}$ de controle.

Por encontrarem-se nessa região, tais pontos levam à suspeita de que o processo possa ter-se deslocado para um estado fora de controle. Neste caso, é razoável tomar-se uma nova amostra de maior tamanho e/ou mais rapidamente. No caso de a estatística ser plotada próximo à linha central, toma-se uma nova amostra de menor tamanho e/ou após um intervalo maior de tempo. Espera-se, assim, reduzir o tempo médio até a sinalização de uma causa especial e o custo médio de inspeção, enquanto o processo estiver sob controle.

Teoricamente, a área entre os limites de controle pode ser dividida em uma região central e múltiplas regiões de advertência. Por exemplo, uma primeira região de advertência (composta de duas sub-regiões, uma acima e outra abaixo da linha central) pode ser formada pela região entre os limites dados por $\mu_{\bar{X}}-1 \sigma_{\bar{X}}$ e $\mu_{\bar{X}}+1 \sigma_{\bar{X}}$, e outros limites de advertência, dados por $\mu_{\bar{X}}-2 \sigma_{\bar{X}}$ e $\mu_{\bar{X}}+2 \sigma_{\bar{X}}$. Uma segunda região de advertência, neste exemplo, pode ser formada pela região entre os limites de advertência $\mu_{\bar{X}}-2 \sigma_{\bar{X}}$ e $\mu_{\bar{X}}+2 \sigma_{\bar{X}}$ e os limites usuais de controle. A região central da carta é a região localizada entre os primeiros limites de advertência. Cada região de advertência corresponde a um conjunto diferente de valores para os parâmetros da carta. Assim, cada vez que um ponto é plotado em uma dessas regiões, uma dentre múltiplas combinações dos valores de $n$ (tamanho da amostra), $v$ (intervalo entre as amostras) e $L$ (coeficiente dos limites de controle) seria escolhida para a seleção da próxima amostra.

O foco deste trabalho são CACs do tipo $\bar{X}$, com intervalo de amostragem adaptativo e tamanho de amostra fixo. Somente uma região de advertência é considerada, localizada a uma distância de $\pm w \sigma_{\bar{X}}$ da linha central. Diversos trabalhos sobre CACs (ver RUNGER \& PIGNATIELLO, 1991, e REYNOLDS, 1995, entre outros) mostraram que o uso de somente 2 conjuntos de valores dos parâmetros $n, v$ e $L$, um valor mínimo e um valor máximo, produz melhores resultados em termos da minimização do tempo médio até a sinalização de uma causa especial; tal simplificação facilita bastante a aplicação prática das CACs. CACs com as características acima são apresentadas em REYNOLDS \& ARNOLD (1989), REYNOLDS (1989), RUNGER \& PIGNATIELLO (1991), RUNGER \& MONTGOMERY (1993), AMIN \& MILLER (1993), REYNOLDS (1995), REYNOLDS et al. (1996) e REYNOLDS (1996a, 1996b).

O funcionamento da carta adaptativa $\bar{X}$ com as características acima pode ser assim descrito. Suponha uma CQ seguindo uma distribuição Normal com parâmetros $\mu$ e $\sigma^{2}$ conhecidos. O valor alvo para a média da CQ é $\mu_{0}$, correspondendo à linha central da carta, e os limites de controle são $\mu_{0} \pm L \sigma / \sqrt{n}$. A área entre os limites de controle é dividida em uma região central, $I_{1}$, e uma região de advertência, $I_{2}$, correspondendo aos intervalos de amostragem $M$ e $m(M>m)$, respectivamente.

A região central $I_{1}$ é formada pelo intervalo $\left(\mu_{0}-w \sigma / \sqrt{n}, \mu_{0}+w \sigma / \sqrt{n}\right)$. A região de advertência $I_{2}$ é formada pela união dos intervalos $\quad\left(\mu_{0}-L \sigma / \sqrt{n}, \mu_{0}-w \sigma / \sqrt{n}\right) \quad \mathrm{e}$ $\left(\mu_{0}+w \sigma / \sqrt{n}, \mu_{0}+L \sigma / \sqrt{n}\right)$. Se a média $\bar{x}_{i}$ da $i$-ésima amostra cair na região $I_{2}$, por exemplo, então a próxima amostra será tomada após um 


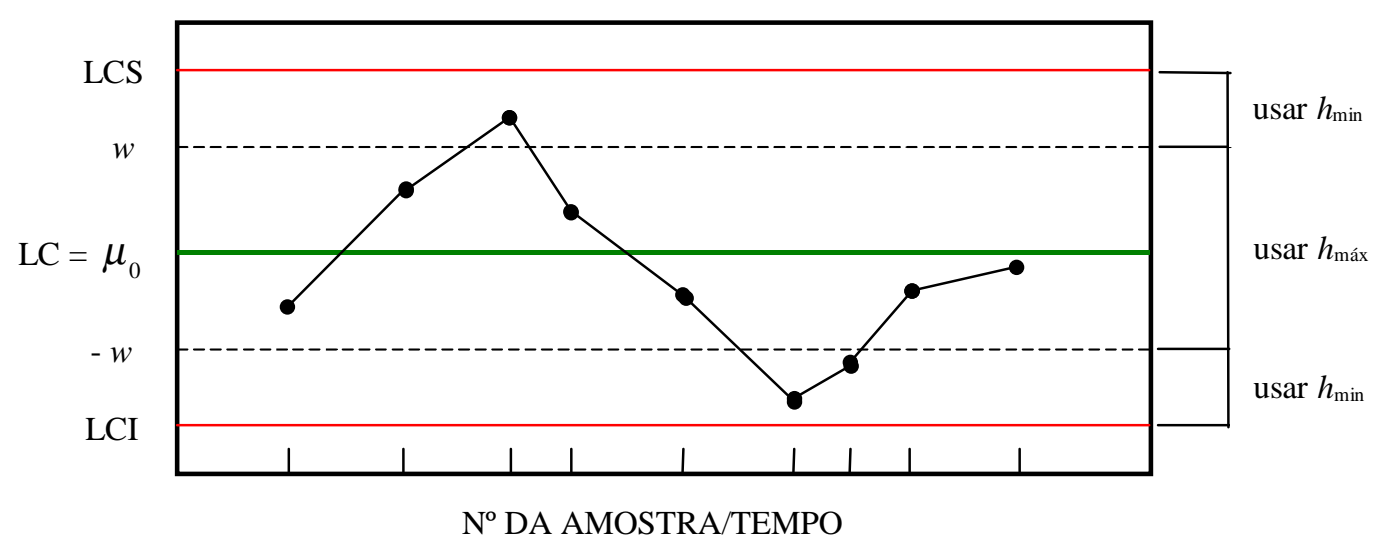

Figura 2 - Exemplo de CAC com intervalo de amostragem adaptativo.

intervalo $m$ de tempo. À parte considerações econômicas, o valor de $m$ é determinado levando-se em conta considerações físicas, como o tempo necessário para se tomar uma amostra; o valor de $M$, por sua vez, deve ser limitado por considerações práticas, como o maior intervalo de tempo que os engenheiros responsáveis pelo processo consideram razoável deixá-lo sem amostragem (digamos, $\theta$ ). Uma carta adaptativa com as características acima está ilustrada na Figura 2.

\section{Um Modelo Econômico para Cartas Adaptativas $\bar{X}$}

$\mathrm{O}$ projeto de CCs consiste na determinação do tamanho de amostra e freqüência de amostragem a ser utilizado no monitoramento da CQ de interesse, bem como na determinação de limites adequados de controle, tendo em vista os erros de tipo I e II admissíveis. Tradicionalmente, este projeto considera apenas aspectos estatísticos. No entanto, os diversos custos associados à utilização das CCs são fortemente influenciados pela escolha de seus parâmetros. Assim, o uso de critérios econômicos no projeto de cartas de controle tem recebido crescente atenção por parte de estatísticos e engenheiros da qualidade (ver MONTGOMERY, 1980, para uma extensa revisão bibliográfica sobre o projeto econômico de $\mathrm{CCs}$ ).
A formulação de um modelo econômico para o projeto de CCs utiliza suposições acerca do comportamento do processo em estudo. A maior parte dos modelos na literatura considera as seguintes suposições (MONTGOMERY, 1996): $\left(s_{1}\right)$ processo possui um único estado sob controle, correspondendo, por exemplo, à média da CQ na ausência de causas especiais; $\left(s_{2}\right)$ processo possui $s \geq 1$ estados fora de controle, cada um associado a um tipo de causa especial; $\left(s_{3}\right)$ causas especiais ocorrem segundo um processo de Poisson, ou seja, o processo permanece sob controle, dado que iniciou sob controle, por um período de tempo exponencialmente distribuído; $\left(s_{4}\right)$ a transição entre os estados é instantânea; e $\left(s_{5}\right)$ o processo não é autocorrigível, só retornando a um estado sob controle após intervenção gerencial.

Os custos associados ao controle dos processos podem ser divididos em três categorias principais: (a) custos de amostragem e medição; (b) custos de investigação e correção de causas especiais; e $(c)$ custos decorrentes da produção de itens não-conformes.

Fazem parte dos custos em (a) as despesas com salários de inspetores e técnicos, custos de testes de equipamentos e custo unitário dos itens amostrados, no caso de testes destrutivos. É comum considerar o custo total dessa categoria como sendo composto por uma parte fixa e outra variável; ou seja, custo total de (a) $=a_{1}+a_{2} n$, 
onde $a_{1}$ representa o custo fixo e $a_{2} n$ representa o custo variável.

A categoria (b) pode ser tratada de diferentes formas. A investigação de alarmes falsos e correção de causas especiais pode consumir diferentes quantidades de recursos. Além disso, cada tipo de causa especial pode ter um custo de correção diferente. Assim, modelos com $s$ estados fora de controle necessitariam de $s+1$ coeficientes de custo (correspondente aos custos de correção de $s$ causas especiais mais o custo de investigação de alarmes falsos). Estes coeficientes devem ser fixados de acordo com o tamanho da mudança ocorrida no processo, pois grandes mudanças implicariam em custos mais altos de reparo (MONTGOMERY, 1996). Essa abordagem pode ser simplificada, reduzindo o nível de detalhamento. Uma abordagem simplificada se justificaria porque, em muitos casos, os custos decorrentes de pequenas e grandes mudanças podem se equiparar (já que pequenas mudanças seriam de difícil localização mas de fácil solução, enquanto grandes mudanças seriam de fácil localização e difícil solução), sendo assim possível utilizar um único coeficiente médio de custo (MONTGOMERY, 1996). No contexto deste trabalho, a localização de uma mudança ocorrida no processo consiste na identificação da causa que a provocou.

Estão incluídos em (c) os custos de refugo ou retrabalho por falhas internas, os custos de troca ou conserto de unidades cobertas por garantia no caso de falhas externas e os custos indiretos por insatisfação dos clientes, que pode levar a uma queda nas vendas futuras do produto ou de outros produtos da empresa.

A partir da definição dos custos envolvidos, os modelos econômicos são formulados de modo que expressem a relação existente entre os custos e os parâmetros da CC. Os modelos partem da premissa de que o funcionamento de um processo produtivo pode ser visto como uma série de ciclos independentes. Cada ciclo inicia com a produção sob controle e continua até que a CC sinalize um estado fora de controle. Neste ponto, é feito um ajuste que deixa o processo novamente sob controle, iniciando-se um novo ciclo. Um modelo econômico pode, assim, ser definido como:

$$
E(A)=E(C) / E(T),
$$

onde: $E(A)$ representa o custo esperado por unidade de tempo, $E(C)$ representa o custo total esperado durante um ciclo e $E(T)$ representa o tamanho esperado de um ciclo. A etapa seguinte no projeto das CCs consiste na aplicação de técnicas de otimização à equação acima, visando determinar seu projeto econômico ótimo.

O modelo econômico proposto neste trabalho para CACs do tipo $\bar{X}$, com intervalo de amostragem adaptativo e tamanho de amostra fixo, é uma adaptação do modelo de DAS et al. (1997), aplicado para o caso de várias causas especiais. Cabe observar que o modelo de DAS et al. (1997) pressupõe que existe somente um tipo de causa especial atuando no processo. Outros modelos, como o de PARK \& REYNOLDS (1994), consideram que o processo está sujeito a múltiplas causas especiais, que ocorrem em tempos que são variáveis aleatórias independentes e exponencialmente distribuídas (com médias dadas por $1 / \lambda_{1}, 1 / \lambda_{2}, 1 / \lambda_{3}, \ldots$ ), produzindo diferentes mudanças na média do processo. Para utilizar o modelo de DAS et al. (1997) quando existem múltiplas causas especiais atuando simultaneamente, como foi feito neste trabalho, pode-se utilizar uma aproximação, considerando-se um mesmo tempo médio $1 / \lambda$ para todas as causas, conforme proposto por DUNCAN (1971) para cartas do tipo $\bar{X}$ com parâmetros fixos.

O modelo é constituído por uma função objetivo e várias restrições, apresentados na sequiência. Também são apresentadas variáveis de decisão e indicadores de desempenho calculados a partir do modelo.

\subsection{Função Objetivo}

O modelo econômico busca minimizar o custo médio do controle de qualidade do sistema 
por unidade de tempo; este custo, designado por $C_{T}$, é dado por DAS et al. (1997):

$$
C_{T}=\frac{1}{T}\left(C_{a}+C_{b}+C_{c}+C_{d}+C_{e}\right),
$$

onde: $T$ é o tempo de ciclo do processo, $C_{a}$ é o custo médio de operação do processo sob controle, $C_{b}$ é o custo médio de operação do processo fora de controle (na presença de causas especiais), $C_{c}$ é o custo médio por ocorrência de alarme falso, $C_{d}$ é o custo médio de localização e reparo de uma causa especial, e $C_{e}$ é o custo médio de amostragem do processo, independentemente do seu estado. Estes elementos são detalhados na sequiência.

Considere um ciclo de processo constituído de quatro fases distintas: $(i)$ sob controle (com ocorrências eventuais de alarmes falsos), (ii) fora de controle, (iii) sob investigação para determinação de causas especiais, e (iv) sofrendo reparo. O tempo de ciclo $T$ é dado por:

$$
\begin{aligned}
T & =1 / \lambda+(1-g)[P(z<-L)+P(z>L)] s T_{f}+ \\
& +A T S_{2}+n E+T_{S}+T_{R}
\end{aligned}
$$

O primeiro termo na equação (4), $1 / \lambda$, corresponde ao tempo médio em que o processo permanece sob controle. Considerando-se a suposição $s_{3}, 1 / \lambda$ corresponde à média de uma variável aleatória que segue uma distribuição exponencial com parâmetro $\lambda$. $\mathrm{O}$ segundo termo na equação (4), $(1-g)[P(z<-L)+P(z>L)] s T_{f}$, considera a ocorrência de alarmes falsos e duas situações possíveis decorrentes: (i) quando $g=1$, o processo continua em funcionamento durante a busca por causas especiais inexistentes; neste caso, o tempo da busca sobrepõe-se ao tempo de operação sob controle, e o termo em questão é cancelado; (ii) quando $g=0$, o processo pára durante a busca por causas especiais inexistentes; neste caso, o tempo de busca, adicionado ao tempo total de ciclo, é dado pelo produto da probabilidade de ocorrência de erro tipo I $[P(z<-L)+P(z>L)]$, do número médio de amostras coletadas do processo sob controle $(s) \mathrm{e}$ do tempo médio de busca quando da ocorrência de um alarme falso $\left(T_{f}\right)$. O número médio de amostras coletadas do processo sob controle $(s)$ é obtido pela equação:

$$
\begin{aligned}
& s=\frac{e^{-\lambda m}}{(1-\Gamma)^{2}}\left\{\left(1-e^{-\lambda M}\right) \frac{P(-w<z<w)}{P(-L<z<L)}+\right. \\
& \left.+\left(1-e^{-\lambda m}\right)\left(\frac{P(-L<z<-w)}{P(-L<z<L)}+\frac{P(w<z<L)}{P(-L<z<L)}\right)\right\}
\end{aligned}
$$

sendo:

$$
\begin{aligned}
\Gamma & =e^{-\lambda M} \frac{P(-w<z<w)}{P(-L<z<L)}+e^{-\lambda m} \frac{P(-L<z<-w)}{P(-L<z<L)}+ \\
& +e^{-\lambda m} \frac{P(w<z<L)}{P(-L<z<L)}
\end{aligned}
$$

O terceiro termo na equação (4), $A T S_{2}$, corresponde ao tempo médio até a ocorrência de um sinal, quando o processo encontra-se fora de controle. O quarto termo na equação (4), $n E$, corresponde ao tempo para coletar uma amostra, executar medições sobre a característica de qualidade de interesse e plotar um ponto na carta. Neste termo, $n$ designa o tamanho da amostra e $E$ corresponde ao tempo médio de processamento por observação amostral. O quinto termo na equação (4), $T_{S}$, corresponde ao tempo médio de busca quando ocorre uma causa especial. Finalmente, o último termo na equação (4), $T_{R}$, corresponde ao tempo médio necessário para reparo da causa especial.

O custo médio de operação do processo sob controle, $C_{a}$, é dado pelo produto entre o custo/hora de produção do processo sob controle $\left(C_{0}\right)$ e o tempo médio em que o processo permanece neste estado $(1 / \lambda)$; isto é,

$$
C_{a}=C_{0} / \lambda
$$

O custo médio de operação do processo fora de controle, $C_{b}$, é dado por

$$
C_{b}=C_{1}\left(A T S_{2}+n E+g T_{S}+h T_{R}\right),
$$


onde: $C_{1}$ é o custo/hora de produção fora de controle e $h$ é uma variável binária, tal que $h=1$ sempre que a produção não for interrompida durante a execução de um reparo; e $h=0$, caso contrário.

$\mathrm{O}$ custo médio devido à ocorrência de alarmes falsos, $C_{c}$, é dado por:

$$
C_{c}=[P(z<-L)+P(z>L)] s Y,
$$

onde $Y$ é o custo médio da ocorrência de um alarme falso.

O custo médio para localizar e reparar uma causa especial, $C_{d}$, é estimado tendo em vista os tipos mais comuns de causas especiais incidentes sobre o processo.

O custo médio de amostragem do processo, $C_{e}$, é dado por:

$$
\begin{aligned}
& C_{e}=\left(a_{1}+a_{2} n\right) s+ \\
& +\left[\left(\frac{a_{1}+a_{2} n}{\gamma}\right)\left(A T S_{2}+n E+g T_{S}+h T_{R}\right)\right],
\end{aligned}
$$

onde: $a_{1}+a_{2} n$ representa o custo associado à coleta, processamento e plotagem de um ponto amostral na carta de controle; e $\gamma$ é o tempo médio entre amostras quando o processo está fora de controle.

\subsection{Restrições}

As restrições propostas para o modelo econômico são listadas a seguir, sendo detalhadas na seqüência.

$$
\begin{aligned}
& m \geq n E \\
& m \leq M \\
& M \leq \theta \\
& w \geq 0 \\
& w \leq L \\
& n \geq \eta \\
& L=3
\end{aligned}
$$

A restrição (11) estabelece como limite inferior do menor intervalo de amostragem o tempo estimado para coletar, medir e plotar uma amostra na CC. A restrição (12) estabelece como limite superior do menor intervalo de amostragem, o maior intervalo de amostragem. A restrição (13) limita em $\theta$ horas o maior intervalo de amostragem, $\operatorname{com} \theta$ estabelecido a partir de considerações práticas. As restrições (14) e (15) estabelecem limites inferior e superior, respectivamente, para o início da região de advertência na CAC. A restrição (16) estabelece um limite inferior $(\eta)$ para o tamanho de amostra, a partir de considerações práticas. Finalmente, a restrição (17) fixa o valor de $L$ em 3 (ver equação 1 ), dado que, na maioria das aplicações de CCs, este é o valor utilizado.

\subsection{Variáveis de Decisão e Indicadores de Desempenho Calculados para o Modelo}

As variáveis de decisão do modelo econômico definem as características da CAC resultante da otimização; são elas: $w, n, L, M$ e $m$ (todas definidas anteriormente). Valores iniciais são atribuídos às variáveis de decisão para dar início à busca de seus valores ótimos que minimizem $o$ custo na equação (3). Como a função objetivo é não-linear, o algoritmo de busca é sensível aos valores iniciais arbitrados para as variáveis de decisão. Assim, diversos valores iniciais devem ser testados em busca da convergência ótima, ou seja, do conjunto de valores para as variáveis de decisão que resulte no menor custo.

Três indicadores de desempenho da CAC têm seu valor determinado a partir dos resultados da otimização (DAS et al., 1997). O primeiro desses indicadores, designado por ANSS, é o número médio de amostras, a partir do momento em que ocorre uma mudança no processo, até a CC sinalizar um estado fora-de-controle, dado por:

$$
A N S S=\frac{1}{P[z<-(L+k \sqrt{n})]+P[z>(L-k \sqrt{n})]},
$$

onde $k$ indica a mudança na média do processo, de $\mu_{0}$ para $\mu_{0}+k \sigma$. 
O segundo indicador, designado por $\mathrm{ATS}_{1}$, é o tempo médio até a $\mathrm{CC}$ sinalizar erroneamente um estado fora-de-controle, resultante de um alarme falso; isto é:

$$
A T S_{1}=\phi /[P(z<-L)+P(z>L)]
$$

Na equação (19), $\phi$ designa o tempo médio entre amostras quando o processo está sob controle, sendo dado por:

$$
\begin{aligned}
& \phi=M \frac{P(-w<z<w)}{P(-L<z<L)}+ \\
& +m \frac{P(-L<z<-w)+P(w<z<L)}{P(-L<z<L)}
\end{aligned}
$$

$\mathrm{O}$ terceiro indicador de desempenho, designado por $\mathrm{ATS}_{2}$, é o tempo médio até a CC sinalizar corretamente um estado fora-decontrole, dado por:

$$
\begin{aligned}
& A T S_{2}=\left[\left(M-\tau_{M}\right) \frac{P(-w<z<w) M}{P(-L<z<L) \phi}\right]+ \\
& +\left[\left(m-\tau_{m}\right) \frac{P(-L<z<-w)+P(w<z<L) m}{P(-L<z<L) \phi}\right]+ \\
& +\gamma(\text { ANSS }-1),
\end{aligned}
$$

onde $\tau_{M}$ designa o tempo médio até a ocorrência de uma mudança na média do processo, dado que ela ocorre dentro do intervalo de amostragem $M$. Considerando-se a suposição $s_{3}$, associada à formulação do modelo, $\tau_{M}$ é igual a (LORENZEN \& VANCE, 1986):

$$
\tau_{M}=\left[1-(1+\lambda M) e^{-\lambda M}\right] /\left[\lambda\left(1-e^{-\lambda M}\right)\right]
$$

Analogamente, $\tau_{m}$ designa o tempo médio de ocorrência de uma mudança na média do processo, dado que ela ocorre dentro do intervalo de amostragem $m$; $\tau_{m}$ é dado por:

$$
\tau_{m}=\left[1-(1+\lambda m) e^{-\lambda m}\right] /\left[\lambda\left(1-e^{-\lambda m}\right)\right]
$$

Finalmente, $\gamma$ representa o tempo médio entre amostras quando o processo está fora-de-controle, considerando-se um desvio da média de $\mu_{0}$ para $\mu_{0}+k \sigma$ (dado em unidades padronizadas):

$$
\begin{gathered}
\gamma=M \frac{P[-(w+k \sqrt{n})<z<(w-k \sqrt{n})]}{P[-(L+k \sqrt{n})<z<(L-k \sqrt{n})]}+ \\
+m \frac{P[(w-k \sqrt{n})<z<(L-k \sqrt{n})]}{P[-(L+k \sqrt{n})<z<(L-k \sqrt{n})]}+ \\
+m \frac{P[-(L+k \sqrt{n})<z<-(w+k \sqrt{n})]}{P[-(L+k \sqrt{n})<z<(L-k \sqrt{n})]}
\end{gathered}
$$

\section{Estudo de Caso}

$\mathrm{O}$ estudo de caso foi realizado em uma indústria de componentes automotivos, localizada na região sul do país. A característica de qualidade selecionada para estudo é a concentricidade de uma peça usinada em uma das operações da empresa. A CQ vinha sendo monitorada por $\mathrm{CCs}$ do tipo $\bar{X}-R$, com parâmetros fixos. A seguir, são apresentados os procedimentos utilizados para a determinação dos valores dos parâmetros, não calculados pelo modelo, que irão compor a função objetivo do modelo econômico.

Para o cálculo do tempo de ciclo $T$ (equação 4), estima-se o tempo médio que o processo permanece sob controle, $1 / \lambda$. Esse tempo foi estimado a partir de CCs $\bar{X}$ arquivadas na empresa, listando-se os tempos entre ocorrências de pontos fora dos limites de controle nas cartas. Todavia, deve-se dar atenção ao fato de que os registros desta empresa armazenavam os pontos fora dos limites da carta, sem diferenciar os alarmes falsos das causas reais de variabilidade, podendo levar a uma estimativa tendenciosa de $1 / \lambda$. O ideal é, sempre que possível, excluir os alarmes falsos para estimar o valor deste tempo.

Esses tempos foram ajustados a uma distribuição exponencial (ver suposição $s_{3}$ ), sendo retirados dois valores espúrios do conjunto de dados. O parâmetro estimado foi $\lambda=1 / 76,308=$ 0,0131 . 
O segundo termo na equação (4) demanda a determinação do valor da variável $g$. No processo estudado, $g=0$, ou seja, o processo pára durante a busca de causas especiais inexistentes. Também é preciso estimar o tempo médio $T_{f}$ de busca quando da ocorrência de um alarme falso. Este tempo depende do procedimento utilizado pelo operador da máquina quando ocorre algum ponto fora dos limites de controle na CC. No presente caso, mesmo que não exista uma causa real provocando o ponto fora dos limites da CC (por ser um alarme falso), o procedimentopadrão utilizado é executar uma limpeza nas castanhas (dispositivos fixadores das peças sendo usinadas). Assim, considerou-se que o tempo de busca quando da ocorrência de um alarme falso é o tempo gasto pelo operador para preparar a máquina para a execução da limpeza nas castanhas. Este tempo, medido junto aos operadores, apresentou uma média de 4 minutos $\left(T_{f}=4 \mathrm{~min}={ }^{4} / 60 h=0,06667 h\right)$.

O tempo necessário desde a coleta da amostra até o registro do ponto na carta, $n E$, também foi medido pela observação do trabalho dos operadores. Para uma amostra de tamanho 3, observou-se um tempo de 40 segundos (isto é, $n E=40 s=0,01111 h$ ). Conseqüentemente, $E={ }^{0,01111} / 3=0,0037037 \mathrm{~h}$.

O processo possui três tipos distintos de causas especiais (suposição $s_{2}$ ), com proporções de ocorrência $p_{i}$ e tempo de busca $b_{i}$. O tempo médio de busca por causas especiais, $T_{S}$, foi estabelecido como sendo a média ponderada entre os três tipos de causas, com ponderação feita por meio dos pesos $p_{i}$. A principal causa especial é a presença de cavacos (pequena lasca resultante do corte da peça) na castanha, resultando em fixação incorreta da peça, com $p_{1}=0,80$ e $b_{1}=4 \min$ (igual ao tempo médio de busca quando da ocorrência de um alarme falso, pois o procedimento utilizado é o mesmo). $\mathrm{O} 2^{\circ}$ tipo de causa mais freqüente é a desregulagem do carregador, dispositivo que coloca a peça na castanha; a freqüência de ocorrência desta causa é $p_{2}=0,15$ e o tempo médio de busca é $b_{2}=6,6667 \mathrm{~min}$. O $3^{\circ}$ tipo de causa é o desgaste da placa, com $p_{3}=0,05$ e $b_{3}=12,16667 \mathrm{~min}$. A estimativa de $T_{S}$ é dada, então, por:

$$
\begin{aligned}
T_{S} & =\frac{4}{60} \cdot 0,80+\frac{6,6667}{60} \cdot 0,15+\frac{12,16667}{60} \cdot 0,05= \\
& =\frac{4,808}{60}=0,080139 \mathrm{~h}
\end{aligned}
$$

Para completar o cálculo de $T$, deve-se determinar o tempo médio gasto no reparo das causas especiais. Novamente, utilizou-se a média ponderada das três principais causas especiais, desta vez analisando-se os tempos de reparo $\left(r_{i}\right)$ ao invés dos tempos de busca. A $1^{\text {a }}$ causa é reparada pela limpeza das castanhas, com tempo médio de reparo dado por $r_{1}=1 \mathrm{~min}$. A $2^{\mathrm{a}}$ causa é reparada pela regulagem do carregador, com $r_{2}=50 \mathrm{~min}$. A $3^{\mathrm{a}}$ causa necessita do torneamento das castanhas para ser reparada, o que implica um tempo médio de reparo de $r_{3}=60 \mathrm{~min}$. $\mathrm{O}$ valor estimado para $T_{R}$ é então:

$$
\begin{aligned}
T_{R} & =\frac{1}{60} \cdot 0,80+\frac{50}{60} \cdot 0,15+\frac{60}{60} \cdot 0,05= \\
& =\frac{11,3}{60}=0,18833 \mathrm{~h}
\end{aligned}
$$

$\mathrm{Na}$ seqüência, estimam-se os custos na equação (3). Os custos $C_{0}$ (custo/hora de produção do processo sob controle) e $C_{1}$ (custo/hora de produção for a de controle) resultam da produção de peças não-conformes, estando o processo sob controle e fora de controle, respectivamente (isto é, custo unitário da peça não-conforme $\times \mathrm{n}^{\circ}$ peças produzidas $/ h \times$ fração de peças não-conformes produzidas sob controle e fora de controle, respectivamente).

Dados históricos mostraram que, da produção total da máquina, cerca de $0,838 \%$ resulta em itens não-conformes, 3,81\% dos quais detectados na própria operação e $96,19 \%$ cerca de cinco operações adiante, onde o valor agregado já é maior. Devido a esta particularidade, o custo unitário de peça não-conforme é de $\$ 3,48$, obtido a partir de uma média ponderada do custo unitário das peças detectadas nas duas situações acima. 
O percentual de peças não-conformes produzidas sob controle foi estimado pelo índice $\mathrm{C}_{p k}$ do processo. $\mathrm{O}$ processo em questão apresenta um $C_{p k}=1,45$, produzindo em torno de 6,81 partes por milhão de não-conformes, ou $0,000681 \%$ de sua produção total (considerandose um limite de especificação unilateral, como é o caso da concentricidade). O percentual de peças não-conformes produzidas fora de controle foi obtido pela diferença entre o percentual total de peças não-conformes e o percentual de peças não-conformes produzidas sob controle $(0,838 \%$ $-0,00068 \%=0,8374 \%)$.

Finalmente, a produção do processo em estudo é 192 peças/ $h$. Com as informações acima, estimaram-se os valores de $C_{0}=\$ 3,48 \times$ $192 \times 0,0000068=\$ 0,004677$ e $C_{1}=\$ 3,48 \times$ $192 \times 0,0083741=\$ 5,5954$. O valor de $h$ na equação (8) foi fixado em $h=0$ (isto é, a produção é interrompida durante a execução de reparos).

O custo médio da ocorrência de um alarme falso, $Y$, na equação (9), decorre da interrupção na produção durante a busca por causas especiais após um alarme falso. No caso, $Y=T_{f} \times$ (custo $/ h$ da máquina parada) $=0,06667 \times \$ 66,14=\$ 4,41$. O mesmo procedimento foi utilizado para calcular o custo médio para localizar e reparar uma causa especial, $C_{d}$, considerando-se os tempos médios de localização e reparo de causas especiais, $T_{S}$ e $T_{R}$. Quando há uma equipe especialmente destinada a realizar o reparo, seu custo deve ser incorporado ao cálculo de $C_{d}$. Aqui, este termo não é incluído, pois o próprio operador da máquina efetua o reparo. Assim, $C_{d}=\left(T_{S}+T_{R}\right) \times($ custo $/ h$ da máquina parada $)=$ $(0,080139+0,18833) \times \$ 66,14=\$ 17,75654$.

Finalmente, estima-se $a_{1}+a_{2} n$, o custo associado à coleta, processamento e plotagem de um ponto amostral na carta de controle. Neste estudo, considera-se o custo fixo de amostragem $\left(a_{1}\right)$ como sendo zero. Além disso, este procedimento não demanda interrupção na produção. Portanto, o custo total de coleta, processamento e plotagem é estimado exclusivamente pelo custo variável correspondente ao salário pago ao operador durante o tempo de execução da tarefa $\left(a_{2} \cdot n=\$ 0,03\right.$, relativo a um tempo $n E=0,011 h)$.

Determinados os valores dos parâmetros de entrada do modelo econômico, utilizou-se um algoritmo de otimização não-linear (Generalized reduced gradient algorithm, proposto por LASDON et al., 1978), disponível na opção Solver do Microsoft EXCEL (1997). O objetivo é minimizar o custo na equação (3), gerando valores ótimos para os parâmetros $w, n, M$ e $m$, sujeito às restrições (11) a (17). Como o resultado ótimo depende da sensibilidade desejada para a CAC na detecção de mudanças na média da CQ de interesse, diferentes valores de $k$ foram utilizados. Para cada $k$, diferentes valores de $w$, $n, M$ e $m$ foram usados para iniciar a otimização, mas nem todos levaram ao mesmo valor ótimo. Os resultados apresentados na Tabela 1 (carta adaptativa) são os valores ótimos globais encontrados. Ou seja, estes resultados representam as estratégias ótimas de controle, no sentido de minimizar o custo, para cada tipo de carta e valor de $k$. Na Tabela 1 também é apresentado o custo médio horário $\left(\mathrm{C}_{\mathrm{CC}}\right)$ associado a diferentes valores de $k$, mediante uma estratégia de controle da qualidade utilizando-se CCs com parâmetros fixos. O modelo econômico usado para obter os valores de $n, m$ e $\mathrm{C}_{\mathrm{CC}}$ está apresentado em LORENZEN \& VANCE (1986).

Observe os valores de $\mathrm{C}_{\mathrm{CAC}}$ e $\mathrm{C}_{\mathrm{CC}}$ para os diferentes valores de $k$; eles indicam o custo médio horário do controle da qualidade no processo em estudo, mediante estratégias adaptativa e tradicional (parâmetros fixos). A CAC apresenta desempenho médio superior no monitoramento do processo se comparada a CC tradicional, conforme evidenciado pelo menor custo médio horário $\mathrm{C}_{\mathrm{CAC}}$ para valores de $k$ inferiores a 2,75; para auxiliar na visualização dos resultados, a Figura 3 traz um gráfico dos custos das cartas CAC e CC como função do valor de $k$.

Em virtude da estratégia das CACs, intensificando-se a freqüência de amostragem a partir da ocorrência de pontos na região de advertência, sua eficiência na detecção de pequenos desvios na média das CQs é bem maior do que aquela 
Tabela 1 - Resultados da otimização e parâmetros resultantes nas CACs e CCs com parâmetros fixos.

\begin{tabular}{|c|c|c|c|c|c|c|c|c|c|c|c|c|}
\hline \multicolumn{10}{|c|}{ Resultados da otimização } \\
\hline \multicolumn{10}{|c|}{ Carta adaptativa } \\
\hline $\boldsymbol{k}$ & $\boldsymbol{w}$ & $\boldsymbol{n}$ & $\boldsymbol{M}$ & $\boldsymbol{m}$ & ANSS & $\mathbf{A T S}_{\mathbf{1}}$ & $\mathbf{A T S}_{\mathbf{2}}$ & $\mathbf{C}_{\mathbf{C A C}}$ & $\boldsymbol{k}$ & $\boldsymbol{n}$ & $\boldsymbol{m}$ & $\mathbf{C}_{\mathbf{C C}}$ \\
\hline \hline 0,5 & 1,404 & 29 & 4,575 & 0,107 & 2,641 & 1432,6 & 3,644 & 0,7587 & 0,5 & 61 & 6,372 & 0,8454 \\
\hline 0,75 & 1,364 & 13 & 3,141 & 0,048 & 2,594 & 968,4 & 2,37 & 0,5765 & 0,75 & 28 & 4,266 & 0,6442 \\
\hline 1 & 1,352 & 7 & 2,43 & 0,028 & 2,53 & 745,1 & 1,768 & 0,4896 & 1 & 16 & 3,231 & 0,5438 \\
\hline 1,25 & 1,353 & 5 & 2,007 & 0,018 & 2,46 & 615,4 & 1,42 & 0,4392 & 1,25 & 10 & 2,62 & 0,4839 \\
\hline 1,5 & 1,361 & 3 & 1,729 & 0,013 & 2,387 & 531,6 & 1,195 & 0,4066 & 1,5 & 7 & 2,218 & 0,4443 \\
\hline 1,75 & 1,528 & 3 & 1,637 & 0,011 & 1,952 & 531,4 & 1,042 & 0,3848 & 1,75 & 5 & 1,937 & 0,4162 \\
\hline 2 & 1,834 & 3 & 1,654 & 0,011 & 1,473 & 573,7 & 0,96 & 0,373 & 2 & 4 & 1,729 & 0,3953 \\
\hline 2,25 & 2,121 & 3 & 1,669 & 0,011 & 1,227 & 598,9 & 0,917 & 0,3663 & 2,25 & 3 & 1,57 & 0,3792 \\
\hline 2,5 & 2,387 & 3 & 1,682 & 0,011 & 1,101 & 614,1 & 0,893 & 0,3623 & 2,5 & 3 & 1,445 & 0,3665 \\
\hline 2,75 & 2,343 & 3 & 1,688 & 0,822 & 1,041 & 619,9 & 0,884 & 0,3593 & 2,75 & 2 & 1,345 & 0,3562 \\
\hline
\end{tabular}

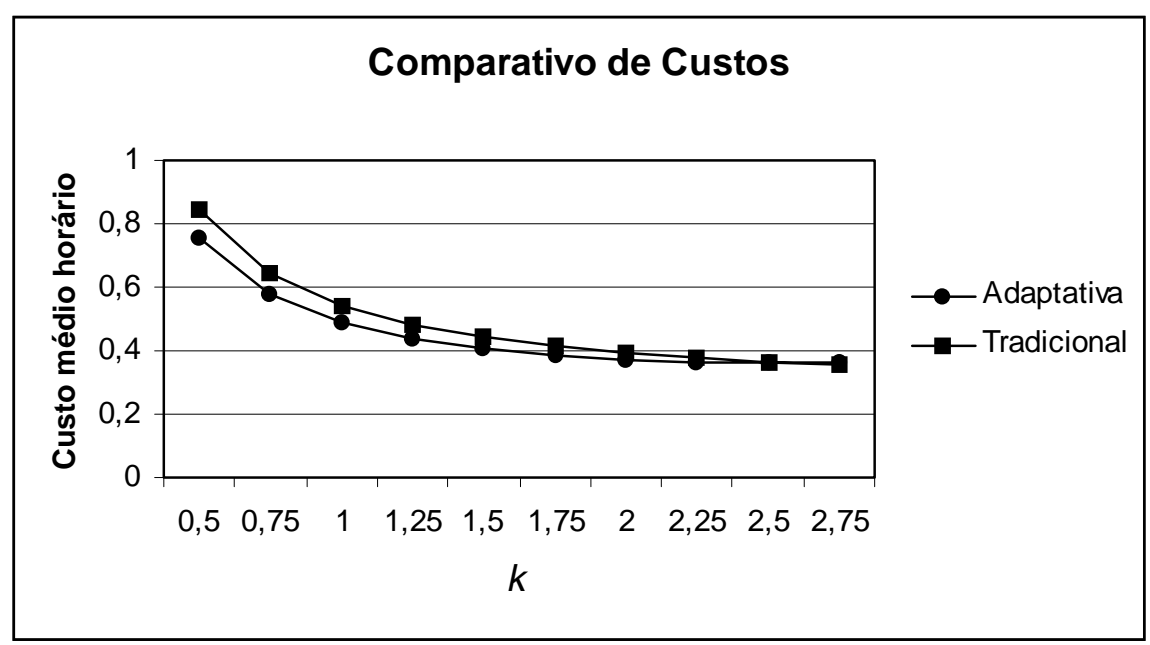

Figura 3 - Custos médios horários nas cartas CAC e CC como função do valor de $k$.

verificada com a utilização das CCs tradicionais. Tal eficiência se reflete em um custo médio inferior incorrido no controle da CQ. Parece evidente que, em termos de custos, a intensificação da frequiência de amostragem sobre o processo é compensada pela redução do tempo necessário para a sinalização de causas especiais, com conseqüente redução da produção de itens não-conformes.

Cabe notar que, embora a diferença entre os custos médios horários das cartas adaptativa e tradicional possa parecer pequena, a economia proporcionada pela carta adaptativa pode ser significativa quando se considera sua utilização por longos períodos de tempo. Além disso, quando utilizadas em várias linhas de produção, a economia poderá ser maior. Uma vez que os resultados obtidos dependem dos parâmetros de entrada, o uso da carta em outros processos, com outros valores para os diversos parâmetros envolvidos, pode proporcionar maiores ganhos econômicos. 


\section{Conclusão}

$\mathrm{N}$ este artigo, propõe-se um modelo econômico para o projeto de cartas adaptativas de controle (CACs) para o monitoramento de médias. O modelo busca determinar parâmetros das CACs que minimizem custos de operação do processo sob controle e fora de controle, custos relacionados à ocorrência de alarmes falsos sobre o processo, custos de localização e reparo de causas especiais, e o custo médio de amostragem do processo. Os parâmetros resultantes da otimização do modelo econômico caracterizam plenamente as cartas adaptativas, definindo a estratégia de monitoramento das características de qualidade representadas nas cartas.

O modelo econômico proposto é aplicado a dados obtidos em um estudo de caso na indústria metal-mecânica. O processo enfocado é o de usinagem de uma peça mecânica e a característica de qualidade estudada, a concentricidade (uma medida dimensional de extrema relevância na determinação da qualidade final da peça). Os resultados da modelagem econômica da carta adaptativa apontam a superioridade desta sobre a CC tradicional na detecção de desvios padronizados na média do processo inferiores a 2,75, a um menor custo médio horário de monitoramento.

\section{Agradecimentos:}

Os autores gostariam de agradecer aos referees anônimos pelo excelente trabalho feito na revisão do presente artigo.

\section{Referências Bibliográficas}

AMIN, R.W.; MILLER, R.W. A Robustness Study of $\bar{X}$ Charts with Variable Sampling Intervals. Journal of Quality Technology, v.25, p.36-44, 1993.

COSTA, A.F.B. Gráficos de Controle $\bar{X}$ para Processos Robustos. Gestão \& Produção, v.5, n.3, p.259-271, 1998 .

DAS, T.K.; JAIN, V.; GOSAVI, A. Economic design of dual sampling interval policies for $\bar{X}$ charts with and without run rules. IIE Transactions, v.29, n.6, p.497-506, 1997.

DUNCAN, A.J. The economic design of $\bar{X}$ charts when there is a multiplicity of assignable causes. Journal of the American Statistical Association, v.66, p.107-121, 1971.

LASDON, L.S.; WAREN, A.D.; JAIN, A.; RATNER, M. Design and testing of a generalized reduced gradient code for nonlinear programming. ACM Trans. Math. Software, v.4, p.34-50, 1978.

LORENZEN, T.J.; VANCE, L.C. The economic design of control charts: a unified approach. Technometrics, v.28, p.3-10, 1996.

MICROSOFT EXCEL. User's Guide, Version 97. Redmond-WA: Microsoft Co., 1997.
MONTGOMERY, D.C. The economic design of control charts: a review and literature survey. Journal of Quality Technology, v.12, p.75-87, 1980.

MONTGOMERY, D.C. Introduction to Statistical Quality Control. 3.ed. New York: John Wiley, 1996.

PARKHIDEH, B.; CASE, K.E. The economic design of a dynamic $\bar{X}$ control chart. IIE Transactions, v.21, p.313-323, 1989.

PARK, C.; REYNOLDS, M.R., JR. Economic Design of a Variable Sample Size $\bar{X}$ Chart. Communications in Statistics - Simulation and Computation, v.23, p.467-83, 1994.

PRABHU, S.S.; MONTGOMERY, D.C.; RUNGER, G.C. Economic-Statistical Design of an Adaptive $\bar{X}$ Chart. International Journal of Production Economics, v.49, p.1-15, 1997.

PROCEP. Manual do Usuário, Ver. 3.01. Porto Alegre: Maxxi Gestão Empresarial, 1999.

REYNOLDS, M.R. Optimal Variable Sampling Interval Control Charts. Sequential Analysis, v.8, p.361-79, 1989. 
REYNOLDS, M.R. Evaluating Properties of Variable Sampling Interval Control Charts. Sequential Analysis, v.14, p.59-97, 1995.

REYNOLDS, M.R. Shewhart and EWMA Variable Sampling Interval Control Charts with Sampling at Fixed Times. Journal of Quality Technology, v.28, n.2, p.199-212, 1996a.

REYNOLDS, M.R. Variable-Sampling-Interval Control Charts with Sampling at Fixed Times. IIE Transactions in Quality and Reliability Engineering, v.28, p.497-510, 1996 b.

REYNOLDS, M.R.; ARNOLD, J.C. Optimal OneSided Shewhart Control Charts with Variable Sampling Intervals. Sequential Analysis, v.8, p.51-77, 1989.
REYNOLDS, M.R.; ARNOLD, J.C.; BAIK, J.W. Variable Sampling Interval $\bar{X}$ Charts in the Presence of Correlation. Journal of Quality Technology, v.28, p.12-30, 1996.

RUNGER, G.C.; MONTGOMERY, D.C. Adaptive Sampling Enhancements for Shewhart Control Charts. IIE Transactions in Quality and Reliability Engineering, v.25, p.41-51, 1993.

RUNGER, G.C.; PIGNATIELLO, J.J. Adaptive Sampling for Process Control. Journal of Quality Technology, v.23, n.2, p.135-55, 1991.

TAGARAS, G. A Survey of Recent Developments in the Design of Adaptive Control Charts. Journal of Quality Technology, v.30, n.3, p.212-231, 1998.

\title{
ECONOMIC DESIGN OF ADAPTIVE CONTROL CHARTS FOR PROCESS MONITORING
}

\begin{abstract}
Statistical control charts have been widely and successfully used for monitoring the performance of industrial processes. Several modifications on the classic Shewhart charts have been proposed in the literature; they aim at providing better tools for monitoring processes with special characteristics. One such modification is the recently developed adaptive control charts. Although providing advantages over Shewhart charts, in particular by signaling out-of-control situations faster, there is no prolific literature on practical implementation of adaptive charts. In this article we propose an economic model for the design of adaptive control charts. We apply the model in a case study from the automotive parts industry, detailing the meaning and determination of model parameters. Adaptive and Shewhart control schemes are compared based on their control costs.
\end{abstract}

Key words: adaptive control charts, control chart economic model, automobile industry, statistical process control. 